\title{
Septins and Bacterial Infection
}

\author{
Vincenzo Torraca and Serge Mostowy * \\ Department of Medicine, MRC Centre of Molecular Bacteriology and Infection, Imperial College London, London, UK
}

Septins, a unique cytoskeletal component associated with cellular membranes, are increasingly recognized as having important roles in host defense against bacterial infection. A role for septins during invasion of Listeria monocytogenes into host cells was first proposed in 2002. Since then, work has shown that septins assemble in response to a wide variety of invasive bacterial pathogens, and septin assemblies can have different roles during the bacterial infection process. Here we review the interplay between septins and bacterial pathogens, highlighting septins as a structural determinant of host defense. We also discuss how investigation of septin assembly in response to bacterial infection can yield insight into basic cellular processes including phagocytosis, autophagy, and mitochondrial dynamics.

Keywords: actin, autophagy, cell-autonomous immunity, cytoskeleton, mitochondria, Listeria, Shigella, septins

\section{INTRODUCTION}

OPEN ACCESS

Edited by:

Manoj B. Menon,

Hannover Medical School, Germany

Reviewed by:

Kenneth G. Campellone,

University of Connecticut, USA

Klaus Aktories,

University of Freiburg, Germany

*Correspondence:

Serge Mostowy

s.mostowy@imperial.ac.uk

Specialty section:

This article was submitted to

Signaling,

a section of the journal

Frontiers in Cell and Developmental

Biology

Received: 31 August 2016

Accepted: 26 October 2016

Published: 11 November 2016

Citation:

Torraca V and Mostowy S (2016)

Septins and Bacterial Infection.

Front. Cell Dev. Biol. 4:127.

doi: 10.3389/fcell.2016.00127
Work has shown that components of the cytoskeleton occupy a central role in innate immunity by promoting bacterial sensing and executing antibacterial functions (Mostowy, 2014; Mostowy and Shenoy, 2015). On the other hand, several intracellular pathogens can exploit the host cytoskeleton for their own advantage to promote invasion, establish a replicative niche, and/or enable dissemination. During infection, some bacteria can invade non-phagocytic cells including epithelial and endothelial cells (Cossart and Sansonetti, 2004; Haglund and Welch, 2011). After entry these pathogens remain enclosed within a membrane-bound compartment or escape to the host cell cytosol (Fredlund and Enninga, 2014). The compartmentalized or cytosolic lifestyle of intracellular pathogens can trigger rearrangements of the cytoskeleton and determine host response to infection. For example, some bacteria that rupture the phagocytic vacuole and escape to the cytosol directly interact with components of the host cytoskeleton to initiate actin-based motility for cell-to-cell spread (Welch and Way, 2013). To counteract bacterial pathogenesis, the cytoskeleton can mediate a variety of cell-autonomous immune defenses, such as activation of the inflammasome (a molecular platform processing inflammatory cytokines) or targeting pathogens to autophagy (a cytosolic degradation process) (Mostowy and Shenoy, 2015).

In comparison to actin, relatively little is known about the role of septins during bacterial infection. Septins are GTP-binding proteins that associate with cellular membrane to form filaments and ring-like structures (Mostowy and Cossart, 2012). They also interact with actin filaments and microtubules, and are therefore considered a cytoskeletal component. Septins were discovered in Saccharomyces cerevisiae as crucial for cell division (Hartwell, 1971). Though septins are highly conserved in fungi and animals, their number across eukaryotic species is variable. There are 7 septins in S. cerevisiae, 2 in Caenorhabditis elegans, 5 in Drosophila melanogaster, at least 17 in zebrafish (Danio rerio), and 13 in both mice and humans (SEPT1-12 and SEPT14). Vertebrate septins can be classified into 4 subgroups, namely the SEPT2, SEPT3, SEPT6, and SEPT7 subgroups, based on homology of sequence and protein domains (Mostowy and Cossart, 2012) (Figure 1A). Septin subunits from the different subgroups interact through their G (consisting 


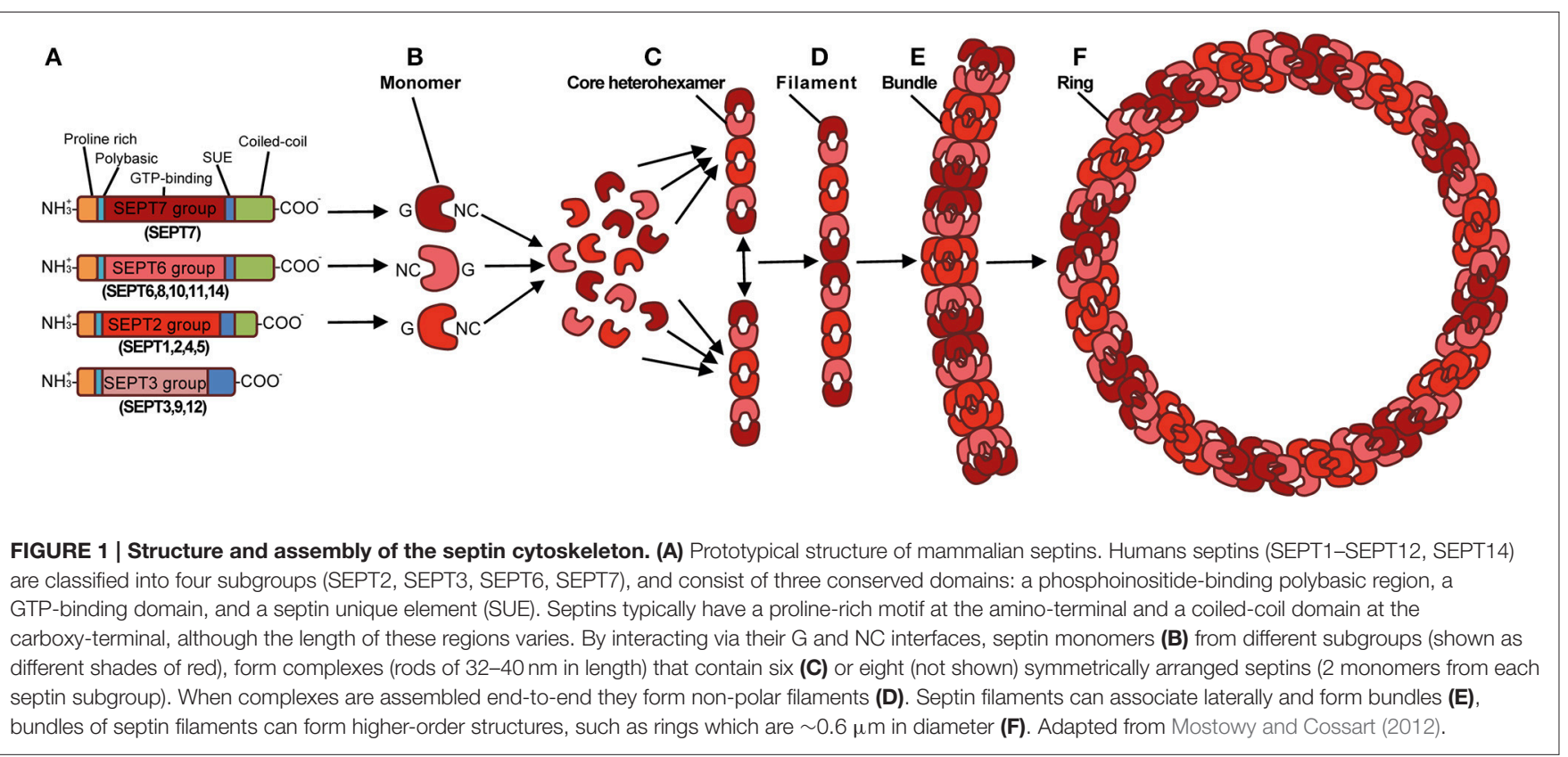

of the GTP-binding domain) and NC (consisting of the aminoand carboxy-terminal regions) interfaces, forming complexes that can join end-to-end to form filaments. Septin filaments can associate, bundle, and go on to form higher-order structures such as rings (Mostowy and Cossart, 2012) (Figures 1B-F).

Septins are involved in numerous biological processes and have been implicated in a wide variety of pathological conditions including cancer, neurodegenerative disorders, and infection. This protein family was first studied in the context of bacterial infection 15 years ago (Pizarro-Cerdá et al., 2002). Identified by mass spectrometry, SEPT9 was associated with the invasion of Listeria monocytogenes into epithelial cells. Since then, septins have been associated with a variety of bacterial pathogens and different stages of the host cell infection process (Mostowy and Cossart, 2012; Krokowski and Mostowy, 2016). Here, we review the literature implicating septins in bacterial pathogenesis (Supplementary Table S1), and discuss how investigation of septin-bacteria interplay can provide novel insights into fundamental processes underlying bacterial infection and also host cell physiology.

\section{SEPTIN FUNCTION IN BACTERIAL ADHESION AND ENTRY INTO HOST CELLS}

Bacterial adhesion to the host cell is fundamental for some pathogens to establish infection. Adhesion prevents the mechanical clearance of extracellular bacteria (e.g., enteropathogenic Escherichia coli and Clostridium difficile) and facilitates the host cell entry of invasive bacteria (e.g., $L$. monocytogenes, Yersinia spp., Shigella flexneri, and Salmonella spp.). In this section, we summarize how several bacterial pathogens manipulate septins at the plasma membrane to support bacterial adherence to the host cell and enable bacterial internalization into both immune and non-immune cells.

\section{Formation of Pedestal-Like Structures during Enteropathogenic Escherichia coli Infection}

Enteropathogenic E. coli (EPEC) is a Gram-negative bacterium responsible for diarrheal disease in humans. By using a type III secretion system (T3SS), a molecular syringe that secretes effector proteins into the host cell, EPEC injects Translocated Intimin receptor (Tir) into the plasma membrane so that it functions as an anchor for Intimin, an effector protein located on the EPEC outer membrane (Croxen et al., 2013). Tir-Intimin interactions mediate the recruitment of Neural Wiskott-Aldrich Syndrome Protein (N-WASP) and the Actin-Related Protein 2/3 (ARP2/3) complex, remodeling of cortical actin filaments, flattening of intestinal microvilli, and ultimately the formation of pedestallike structures raising the bacteria above the plasma membrane (Croxen et al., 2013; Lai et al., 2013).

A recent study discovered that EPEC infection phosphorylates SEPT9 in a T3SS-dependent manner (Scholz et al., 2015). The depletion or impaired phosphorylation of SEPT9 reduced adherence of EPEC to the host cells and also EPEC-mediated cytotoxicity. How septins and their phosphorylation contribute to EPEC adhesion remains to be established. Work has shown an important role for septin phosphorylation in higher-order assembly into rings (Dobbelaere et al., 2003; Kinoshita, 2003; Hernández-Rodríguez and Momany, 2012; Meseroll et al., 2013). Given that septins are involved in actin dynamics, septin assembly may promote actin rearrangements and membrane dynamics which mediate pedestal biogenesis (Scholz et al., 2015) (Figure 2A). The T3SS effector that mediates SEPT9 

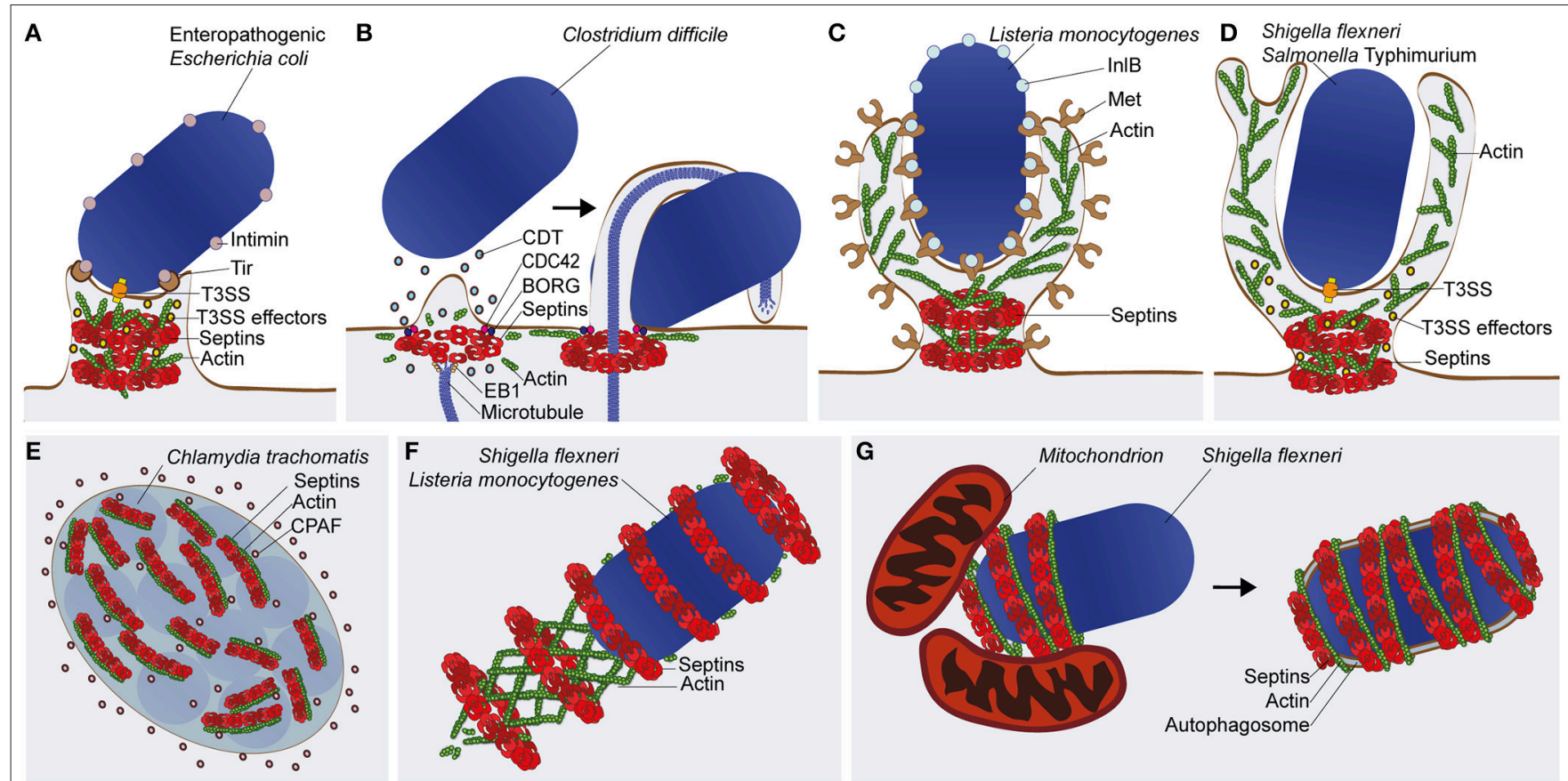

FIGURE 2 | Septin recruitment and function in bacterial infections. (A) The enteropathogenic E. coli (EPEC) pedestal. Type 3 secretion system (T3SS) effectors of EPEC are implicated in septin phosphorylation and assembly into filaments and rings. It is proposed that septins assemblies (depicted here as rings) remodel cortical actin for pedestal biogenesis (B) The C. difficile microtubule-based protrusion. Intoxication with the C. difficile toxin (CDT) leads to patches of cortical actin/septin depolymerization followed by rearrangement of septins into ring-like structures, facilitated by the factors Cell Division Control Protein 42 (CDC42) and Binder of Rho GTPase (BORG). By interacting with End Binding 1 (EB1) protein, septins redirect microtubule polymerization and initiate protrusion formation. Protrusions prevent the mechanical clearance of $C$. difficile by wrapping around the bacterium. (C) Zipper-mediated entry by $L$. monocytogenes. During invasion of Listeria into host cells, interaction of the virulence factor Internalin B (InIB) with its membrane receptor Met, initiates septin recruitment to the entry site where it forms ring-like structures at the base of the phagocytic cup. (D) Trigger-mediated entry by S. flexneri or S. Typhimurium. Septin ring-like structures associate with the bacteria upon injection of T3SS effectors into the host cell to induce membrane ruffles and macropinocytosis at the bacterial entry site. In the case of (C,D), septins at the plasma membrane may facilitate actin remodeling, membrane protrusion, and bacterial internalization by acting as diffusion barriers for lipids and signaling molecules. (E) The C. trachomatis inclusion vacuole. Septin and actin filaments form a coat that associate with the cytosolic surface of the inclusion vacuole, and contributes to its remodeling to accommodate bacterial growth. The chlamydial enzyme CPAF is able to cleave septins for control of inclusion remodeling. (F) The $L$. monocytogenes or S. flexneri actin tail. Although dispensable for actin-based motility, septins assemble into rings around a subset of cytosolic bacteria polymerizing actin tails. The precise role of septins in the functionality of the actin tail remains unknown. (G) The S. flexneri septin cage. Septins assemble into cage-like structures around a subset of cytosolic bacteria polymerizing actin. In the case of Shigella, septin cage assembly is promoted by mitochondria and leads to the restriction of bacterial replication by autophagy.

phosphorylation remains unknown, however a previous study performed in S. cerevisiae showed that overexpression of the T3SS effector proteins Mitochondrial associated protein (Map) and $E$. coli secretion protein $\mathrm{F}(\mathrm{EspF})$ cause abnormal and mislocalized septin assemblies that affect morphogenesis and cell division (Rodríguez-Escudero et al., 2005). The same study implicated Map and EspF in the activation of a phosphorylation cascade via the Mitogen-Activated Protein Kinase (MAPK) pathway. Taken together, it is tempting to speculate that EPEC virulence factors activate a phosphorylation cascade to mediate the recruitment and assembly of septins at the plasma membrane for actin-mediated pedestal formation. Further experiments will be required to address this hypothesis. Notably, increased phosphorylation of SEPT9 has also been identified from infections with other T3SS-positive enterobacteria, including Shigella and Salmonella spp. (Rogers et al., 2011; Schmutz et al., 2013), raising the possibility that septin phosphorylation is commonly exploited by bacterial pathogens to coordinate rearrangements of the actin cytoskeleton and plasma membrane.

\section{Microtubule-Based Protrusions from Clostridium difficile Infection}

Hypervirulent strains of $C$. difficile infect humans provoking colitis, antibiotic-associated diarrhea, and occasionally death. The increased virulence of some $C$. difficile isolates is associated with the expression of the $C$. difficile transferase (CDT), a toxin that mediates formation of microtubule-based protrusions, which wrap around bacteria and increase their adherence to the host cell (Schwan et al., 2014). When exposing cells to CDT, the cellular cortex undergoes extensive remodeling, resulting in patches of actin depolymerization. Complexes of SEPT2SEPT6-SEPT7-SEPT9 are recruited to the plasma membrane shortly after initial depolymerization events and form collarlike structures that initiate CDT-dependent protrusions (Nölke et al., 2016) (Figure 2B). Depletion of septins by siRNA or their 
inhibition with forchlorfenuron reduced protrusion formation, while septin overexpression increased protrusion emergence. At the protrusion base, septins interact with the GTPase Cell Division Control Protein 42 (CDC42) and its effector Binder of Rho GTPase (BORG) (Nölke et al., 2016). These results are consistent with previous work using non-infected HeLa cells showing the regulation of septin assembly by BORGs (Joberty et al., 2001).

Septins have high affinity to End Binding 1 (EB1), a protein which associates to the plus end of microtubules, suggesting that septins guide microtubule polymerization in the extending protrusion (Nölke et al., 2016). The discovery that septins mediate microtubule apical guidance can have broad implications in cell biology. Septins are well known to localize at the base of the primary cilium, a membrane-delimited eukaryotic appendage also sustained by a microtubule-based scaffold that senses and transduces a variety of extracellular stimuli $(\mathrm{Hu}$ et al., 2010). Work has shown that depletion of SEPT2 results in mislocalization of ciliary membrane proteins and impairs ciliogenesis ( $\mathrm{Hu}$ et al., 2010). Together, these results indicate that septins play a key role in maintenance of membrane compartmentalization and work as a membrane diffusion barrier, a function also attributed to septin rings at the mother-bud neck in S. cerevisiae (Barral et al., 2000; Takizawa et al., 2000; Caudron and Barral, 2009).

\section{"Zipper" and "Trigger"-Mediated Entry of Invasive Bacteria}

To enter non-phagocytic cells, invasive bacteria have different mechanisms to manipulate host signaling pathways leading to their uptake. Some bacteria, such as L. monocytogenes and Yersinia spp., can enter host cells via a mechanism called "zippering" that is activated via the direct interaction between bacterial surface components and host cell receptors at the plasma membrane. Other bacteria, such as $S$. flexneri and Salmonella enterica serovar Typhimurium, enter non-phagocytic cells by a mechanism called "triggering" that is dependent on the injection of T3SS effector proteins to stimulate host cell membrane ruffling and engulfment via a macropinocytosis-like process.

L. monocytogenes, a Gram-positive bacterium and foodborne pathogen, can stimulate its internalization into host cells via interactions between its surface proteins Internalin (InlA) and Internalin B (InlB) with plasma membrane proteins E-cadherin and Met, respectively (Pizarro-Cerdá et al., 2012). To discover other proteins required for Listeria entry, proteomic analysis was performed and identified enrichment of SEPT9 at the entry site during InlB-mediated invasion (Pizarro-Cerdá et al., 2002). Follow-up work revealed that septin collar-like structures assembled in response to actin polymerization at sites of Listeria entry (Mostowy et al., 2009b) (Figure 2C). Interestingly, SEPT2 promotes Listeria invasion while SEPT11 restricts it, suggesting different roles for different septins in bacterial entry (Mostowy et al., 2009a,b, 2011a; Kühbacher et al., 2015). Septins also form collar-like structures at sites of entry of "triggering" bacteria, such as S. flexneri and S. Typhimurium (Mostowy et al., 2009b) (Figure 2D). Here, septins may additionally facilitate membrane fusion events during the bacteria-induced macropinosome trafficking (Dolat and Spiliotis, 2016; Weiner et al., 2016). Together, this highlights septin assembly as a general response to actin-mediated bacterial invasion. In addition to the above studies performed using infection of non-phagocytic host cells, work has shown that SEPT2 and SEPT11 assemble into collar-like structures at the base of the phagocytic cup in macrophages and neutrophils to enable phagocytosis (Huang et al., 2008).

The precise role of septins in mediating bacterial entry remains to be established. Considering their function in compartmentalizing plasma membrane (Kusumi et al., 2012; Bridges and Gladfelter, 2015), septins may recruit host cell receptors, phospholipids, and/or signaling molecules to orchestrate the cytoskeletal rearrangements and membrane extension which underpin formation of the phagocytic cup (Barral et al., 2000; Caudron and Barral, 2009; Mostowy and Cossart, 2011; Ostrowski et al., 2016).

\section{SEPTIN INTERACTIONS WITH INTRACELLULAR BACTERIAL PATHOGENS}

After phagocytosis and entry into host cells, bacteria can adopt different intracellular lifestyles. Some invasive pathogens, such as Chlamydia trachomatis and Legionella pneumophila, can remodel their phagocytic compartment to establish an intracellular niche for persistence and replication. In contrast, other bacterial pathogens such as L. monocytogenes, S. flexneri, Mycobacterium marinum, and Rickettsia spp., can escape from the phagocytic vacuole to invade the host cell cytosol (Ray et al., 2009). In the following section, we discuss the interactions between septins and intracellular bacteria. We focus on septin roles in maintenance of the C. trachomatis intracellular niche, and also in the compartmentalization of cytosolic $S$. flexneri for host defense.

\section{Septin Interactions with Chlamydia trachomatis}

C. trachomatis is an obligate intracellular bacterium that replicates inside an inclusion vacuole delimited by host membranes. The parasitic lifecycle of Chlamydia relies on the recruitment of actin and vimentin intermediate filaments to the inclusion (Kumar and Valdivia, 2008). To remodel the cytoskeleton, C. trachomatis secretes the protease Chlamydial proteasome-like activity factor (CPAF) into the host cell cytosol and increases the size/flexibility of the inclusion to enable bacterial replication (Kumar and Valdivia, 2008). A recent study revealed that complexes of SEPT2-SEPT11SEPT7-SEPT9 are also recruited to the cytosolic surface of the Chlamydia inclusion, where septins associate with actin filaments (Volceanov et al., 2014) (Figure 2E). Inhibition of septin assembly using forchlorfenuron reduced the expansion of inclusion vacuoles. Additionally, depletion of septins by siRNA abolished the formation of septin/actin coats around the Chlamydia inclusion and restricted the extrusion of intact 
inclusions from host cells. The same study revealed that SEPT2 is a host cell substrate for CPAF, like vimentin. While the exact role of SEPT2-CPAF interactions awaits investigation, it has been suggested that cleavage of SEPT2 by CPAF contributes to dynamic remodeling of the cytoskeleton around the inclusion.

\section{A Role for Septins in Actin Tail Polymerization?}

To evade cytosolic immunity, some bacteria that escape from the phagocytic vacuole can polymerize actin for actin-based motility and cell-to-cell dissemination (Welch and Way, 2013). Interestingly, bacteria (including Listeria spp., Shigella spp., $M$. marinum, Burkholderia spp., and Rickettsia spp.) have evolved different mechanisms to polymerize actin tails (Gouin et al., 2005; Welch and Way, 2013). Just as septins are recruited to actin polymerization at the plasma membrane, septins are also recruited to some actin-polymerizing bacteria in the cytosol as ring-like structures (Mostowy et al., 2010) (Figure 2F). Although work using septin-depleted cells failed to reveal any role for septins in the functionality of actin tails (Mostowy et al., 2010), it is possible that septins impact actin tails in aspects difficult to detect using transient depletion techniques in tissue culture cells. Given that both actin-based motility and septin assembly can be reconstituted using purified proteins in cell-free conditions (Loisel et al., 1999; Bridges and Gladfelter, 2016), it seems likely that biochemical reconstitution systems will be of great value to elucidate whether septins have a role in the functionality of actin tails.

\section{Septin Interplay with Bacterial Autophagy}

Septins can recognize some bacterial pathogens that escape to the host cell cytosol and polymerize actin, forming cage-like structures to entrap bacteria and prevent actin tail formation (Mostowy et al., 2010) (Figure 2G). Indeed, the frequency of actin tails is significantly enhanced in septin-depleted cells infected with bacteria normally recognized by septin cages. Recent work has also shown that septin cages assemble to restrict bacterial replication (Sirianni et al., 2016). Septin cages have been observed for both $S$. flexneri and M. marinum during infection of tissue culture cells in vitro and during infection of zebrafish in vivo (Mostowy et al., 2010, 2013; Sirianni et al., 2016). Collectively, these observations highlight septin caging of cytosolic bacteria as an evolutionarily-conserved assembly valuable for host defense (Mostowy, 2014; Mostowy and Shenoy, 2015).

Shigella entrapped in septin cages are also targeted to autophagy (Mostowy et al., 2010; Sirianni et al., 2016) (Figure 2G). Autophagy is a membrane trafficking process that can redirect cytosolic material, including bacteria, to lytic compartments (Levine et al., 2011; Mostowy, 2014). A group of autophagy-related (ATG) proteins constitute the core machinery responsible for the formation, elongation, and maturation of the autophagosome. In the case of bacterial autophagy, ubiquitination and its recognition by a set of ubiquitin-binding autophagy receptors (e.g., p62/SQSTM1, NDP52/CALCOCO2), can direct the ATG machinery around bacterial cargo (Khaminets et al., 2016; Maculins et al., 2016). Strikingly, the recruitment of septins and autophagy markers are tightly connected. The depletion of septins (SEPT2, SEPT7, or SEPT9) or autophagy components (p62, NDP52, ATG5, ATG6, or ATG7) abrogates both septin cage assembly and bacterial autophagy (Mostowy et al., 2010, 2011b; Sirianni et al., 2016). These data suggest that, at least during autophagy of Shigella, septin cage assembly and autophagosome formation are interdependent (Mostowy and Cossart, 2012). It is interesting to consider that septin cages have been observed during autophagy of S. flexneri and $M$. marinum, distinct bacterial pathogens that both polymerize actin via the recruitment of N-WASP (Mostowy et al., 2010, 2013; van der Vaart et al., 2014). In contrast, septin cages have not been observed for Listeria spp. (Mostowy et al., 2010), partly because cytosolic Listeria polymerize actin tails via ActA, a bacterial effector that mimics host cell N-WASP and prevents the ubiquitination and recognition of bacteria by autophagy (Yoshikawa et al., 2009; Mostowy et al., 2011b). How exactly septins and autophagy are recruited to bacteria remains to be established. Recent work in S. cerevisiae has shown that septins are involved in the early stages of autophagosome formation during starvation (Barve et al., 2016). Collectively, these data suggest that septins have a fundamental role in multiple pathways of autophagy, including starvation-induced autophagy and bacterial autophagy.

To better understand the molecules and events required for septin cage assembly, proteomic analysis of the Shigella-septin cage was performed (Sirianni et al., 2016). From this approach, 56 host proteins were identified to interact with septins in Shigellainfected conditions, including p62 and the ATG8 family member Light Chain 3 B (LC3B). Surprisingly, proteomics also revealed that $21.4 \%$ of septin cage-associated proteins were annotated as exclusively mitochondrial. Indeed, high-resolution microscopy showed that mitochondria support septin assembly into the cages that entrap Shigella for autophagy. A role for mitochondria in septin cage assembly was confirmed by depletion of factors implicated in mitochondria fission and fusion (Sirianni et al., 2016). The depletion of Dynamin Related Protein 1 (DRP1), which elongates mitochondria and increases their availability as a membrane source for septin assembly, significantly increased septin caging. Conversely, the depletion of Mitofusin 1 (MFN1), which fragments mitochondria and limits their availability as a membrane source for septin assembly, significantly reduced septin caging. Shigella invasion is well known to induce mitochondria fragmentation (Carneiro et al., 2009; Lum and Morona, 2014). Remarkably, fragmentation of mitochondria enables Shigella to escape from septin cages and autophagy recognition (Sirianni et al., 2016). L. monocytogenes, which avoids septin caging, can induce fragmentation of mitochondria via Listeriolysin O (LLO), a pore-forming toxin (Stavru et al., 2011). Together, these observations suggest that fragmentation of mitochondria may be a general mechanism for bacterial pathogens to escape from cell-autonomous immunity (Sirianni et al., 2016).

Important studies have shown that septins recognize micron scale curvature at the plasma membrane, and that septin assembly is membrane-facilitated (Tanaka-Takiguchi et al., 2009; Bridges and Gladfelter, 2015; Bridges et al., 2016). In 
agreement with this, septin assemblies are closely associated with invaginations and protrusions of the plasma membrane including the phagocytic cup, the cleavage furrow, and the base of dendritic spines and cilia (Bridges et al., 2016; LobatoMárquez and Mostowy, 2016). In contrast, septin association with sources of membrane in the cytosol remains to be established. New studies have shown that septins interact with mitochondria and enable mitochondrial fission, in a process called mitokinesis (Pagliuso et al., 2016; Sirianni et al., 2016). Strikingly, septin function at the plasma membrane (e.g., phagocytosis, cytokinesis) and in the cytosol (e.g., septin caging, mitokinesis) all involve actin and non-muscle myosin II (Mostowy and Cossart, 2012; Sirianni et al., 2016). Next, it will be important to investigate different sources of cytosolic membrane (e.g., mitochondria, ER), and their precise role in septin assembly.

\section{CONCLUSIONS}

Investigation into the cell biology of bacterial infection has significantly improved our understanding of both infection and cytoskeleton biology (Haglund and Welch, 2011). For example, the study of actin-tail formation by cytosolic bacteria has enabled major discoveries underlying bacterial pathogenesis and actin biology (Welch and Way, 2013). In this review, we have discussed several examples of how investigation of septin assembly in response to bacterial invasion is discovering novel aspects of septin biology and cell-autonomous immunity. Our current knowledge of septin function during infection mostly derives from work performed in vitro using non-phagocytic cells (e.g.,

\section{REFERENCES}

Barral, Y., Mermall, V., Mooseker, M. S., and Snyder, M. (2000). Compartmentalization of the cell cortex by septins is required for maintenance of cell polarity in yeast. Mol. Cell 5, 841-851. doi: 10.1016/S1097-2765(00)80324-X

Barve, G., Sridhar, S., Aher, A., Singh, S., Lakshmeesha, K. N., and Manjithaya, R. (2016). Septins are Involved at the Early Stages of Macroautophagy. bioRxiv.

Bridges, A. A., and Gladfelter, A. S. (2015). Septin form and function at the cell cortex. J. Biol. Chem. 290, 17173-17180. doi: 10.1074/jbc.R114.634444

Bridges, A. A., and Gladfelter, A. S. (2016). In vitro reconstitution of septin assemblies on supported lipid bilayers. Methods Cell Biol. 136, 57-71. doi: 10.1016/bs.mcb.2016.03.025

Bridges, A. A., Jentzsch, M. S., Oakes, P. W., Occhipinti, P., and Gladfelter, A. S. (2016). Micron-scale plasma membrane curvature is recognized by the septin cytoskeleton. J. Cell Biol. 213, 23-32. doi: 10.1083/jcb.201512029

Carneiro, L. A., Travassos, L. H., Soares, F., Tattoli, I., Magalhaes, J. G., Bozza, M. T., et al. (2009). Shigella induces mitochondrial dysfunction and cell death in nonmyleoid cells. Cell Host Microbe 5, 123-136. doi: 10.1016/j.chom.2008.12.011

Caudron, F., and Barral, Y. (2009). Septins and the lateral compartmentalization of eukaryotic membranes. Dev. Cell 16, 493-506. doi: 10.1016/j.devcel.2009.04.003

Cossart, P., and Sansonetti, P. J. (2004). Bacterial invasion: the paradigms of enteroinvasive pathogens. Science 304, 242-248. doi: 10.1126/science.1090124

Croxen, M. A., Law, R. J., Scholz, R., Keeney, K. M., Wlodarska, M., and Finlay, B. B. (2013). Recent advances in understanding enteric pathogenic Escherichia coli. Clin. Microbiol. Rev. 26, 822-880. doi: 10.1128/CMR.00022-13
HeLa). Considering the emerging roles for the host cytoskeleton in cell-autonomous immunity (Mostowy and Shenoy, 2015), future work should address septin function in immune cells. More insight into septin roles in host defense can also come from the use of animal models. Indeed, septin-bacteria interactions can be studied using zebrafish infection models innovated to study the cell biology of infection in vivo (Mostowy et al., 2013; Willis et al., 2016). We propose that use of in vivo systems, including the zebrafish and mouse, will be essential to illuminate a more complete understanding of septin biology during host defense against bacterial infection.

\section{AUTHOR CONTRIBUTIONS}

VT and SM jointly wrote the manuscript.

\section{ACKNOWLEDGMENTS}

VT is supported by a Marie Skłodowska-Curie Individual Fellowship (H2020-MSCA-IF-2015 - 700088). Work in SM laboratory is supported by a Wellcome Trust Research Career Development Fellowship (WT097411MA) and the Lister Institute of Preventive Medicine.

\section{SUPPLEMENTARY MATERIAL}

The Supplementary Material for this article can be found online at: http://journal.frontiersin.org/article/10.3389/fcell. 2016.00127/full\#supplementary-material

Dobbelaere, J., Gentry, M. S., Hallberg, R. L., and Barral, Y. (2003). Phosphorylation-dependent regulation of septin dynamics during the cell cycle. Dev. Cell 4, 345-357. doi: 10.1016/S1534-5807(03)00061-3

Dolat, L., and Spiliotis, E. T. (2016). Septins promote macropinosome maturation and traffic to the lysosome by facilitating membrane fusion. J. Cell Biol. 214, 517-527. doi: 10.1083/jcb.201603030

Fredlund, J., and Enninga, J. (2014). Cytoplasmic access by intracellular bacterial pathogens. Trends Microbiol. 22, 128-137. doi: 10.1016/j.tim.2014.01.003

Gouin, E., Welch, M. D., and Cossart, P. (2005). Actin-based motility of intracellular pathogens. Curr. Opin. Microbiol. 8, 35-45. doi: 10.1016/j.mib.2004.12.013

Haglund, C. M., and Welch, M. D. (2011). Pathogens and polymers: microbehost interactions illuminate the cytoskeleton. J. Cell Biol. 195, 7-17. doi: $10.1083 /$ jcb. 201103148

Hartwell, L. H. (1971). Genetic control of the cell division cycle in yeast. IV. Genes controlling bud emergence and cytokinesis. Exp. Cell Res. 69, 265-276. doi: 10.1016/0014-4827(71)90223-0

Hernández-Rodríguez, Y., and Momany, M. (2012). Posttranslational modifications and assembly of septin heteropolymers and higher-order structures. Curr. Opin. Microbiol. 15, 660-668. doi: 10.1016/j.mib.2012. 09.007

Hu, Q., Milenkovic, L., Jin, H., Scott, M. P., Nachury, M. V., Spiliotis, E. T., et al. (2010). A septin diffusion barrier at the base of the primary cilium maintains ciliary membrane protein distribution. Science 329, 436-439. doi: 10.1126/science. 1191054

Huang, Y. W., Yan, M., Collins, R. F., Diciccio, J. E., Grinstein, S., and Trimble, W. S. (2008). Mammalian septins are required for phagosome formation. Mol. Biol. Cell 19, 1717-1726. doi: 10.1091/mbc.E07-07-0641 
Joberty, G., Perlungher, R. R., Sheffield, P. J., Kinoshita, M., Noda, M., Haystead, T., et al. (2001). Borg proteins control septin organization and are negatively regulated by Cdc42. Nat. Cell Biol. 3, 861-866. doi: 10.1038/ncb1001-861

Khaminets, A., Behl, C., and Dikic, I. (2016). Ubiquitin-dependent and independent signals in selective autophagy. Trends Cell Biol. 26, 6-16. doi: 10.1016/j.tcb.2015.08.010

Kinoshita, M. (2003). Assembly of mammalian septins. J. Biochem. 134, 491-496. doi: $10.1093 / \mathrm{jb} / \mathrm{mvg} 182$

Krokowski, S., and Mostowy, S. (2016). Investigation of septins using infection by bacterial pathogens. Methods Cell Biol. 136, 117-134. doi: 10.1016/bs.mcb.2016.03.018

Kühbacher, A., Emmenlauer, M., Rämo, P., Kafai, N., Dehio, C., Cossart, P., et al. (2015). Genome-wide siRNA screen identifies complementary signaling pathways involved in listeria infection and reveals different actin nucleation mechanisms during listeria cell invasion and actin comet tail formation. MBio 6, e00598-00515. doi: 10.1128/mBio.00598-15

Kumar, Y., and Valdivia, R. H. (2008). Actin and intermediate filaments stabilize the Chlamydia trachomatis vacuole by forming dynamic structural scaffolds. Cell Host Microbe 4, 159-169. doi: 10.1016/j.chom.2008.05.018

Kusumi, A., Fujiwara, T. K., Chadda, R., Xie, M., Tsunoyama, T. A., Kalay, Z., et al. (2012). Dynamic organizing principles of the plasma membrane that regulate signal transduction: commemorating the fortieth anniversary of Singer and Nicolson's fluid-mosaic model. Annu. Rev. Cell Dev. Biol. 28, 215-250. doi: 10.1146/annurev-cellbio-100809-151736

Lai, Y., Rosenshine, I., Leong, J. M., and Frankel, G. (2013). Intimate host attachment: enteropathogenic and enterohaemorrhagic Escherichia coli. Cell. Microbiol. 15, 1796-1808. doi: 10.1111/cmi.12179

Levine, B., Mizushima, N., and Virgin, H. W. (2011). Autophagy in immunity and inflammation. Nature 469, 323-335. doi: 10.1038/nature09782

Lobato-Márquez, D., and Mostowy, S. (2016). Septins recognize micron-scale membrane curvature. J. Cell Biol. 213, 5-6. doi: 10.1083/jcb.201603063

Loisel, T. P., Boujemaa, R., Pantaloni, D., and Carlier, M. F. (1999). Reconstitution of actin-based motility of Listeria and Shigella using pure proteins. Nature 401, 613-616. doi: 10.1038/44183

Lum, M., and Morona, R. (2014). Dynamin-related protein Drp1 and mitochondria are important for Shigella flexneri infection. Int. J. Med. Microbiol. 304, 530-541. doi: 10.1016/j.ijmm.2014.03.006

Maculins, T., Fiskin, E., Bhogaraju, S., and Dikic, I. (2016). Bacteria-host relationship: ubiquitin ligases as weapons of invasion. Cell Res. 26, 499-510. doi: $10.1038 /$ cr.2016.30

Meseroll, R. A., Occhipinti, P., and Gladfelter, A. S. (2013). Septin phosphorylation and coiled-coil domains function in cell and septin ring morphology in the filamentous fungus Ashbya gossypii. Eukaryotic Cell 12, 182-193. doi: 10.1128/EC.00251-12

Mostowy, S. (2014). Multiple roles of the cytoskeleton in bacterial autophagy. PLoS Pathog. 10:e1004409. doi: 10.1371/journal.ppat.1004409

Mostowy, S., Bonazzi, M., Hamon, M. A., Tham, T. N., Mallet, A., Lelek, M., et al. (2010). Entrapment of intracytosolic bacteria by septin cage-like structures. Cell Host Microbe 8, 433-444. doi: 10.1016/j.chom.2010.10.009

Mostowy, S., Boucontet, L., Mazon Moya, M. J., Sirianni, A., Boudinot, P., Hollinshead, M., et al. (2013). The zebrafish as a new model for the in vivo study of Shigella flexneri interaction with phagocytes and bacterial autophagy. PLoS Pathog. 9:e1003588. doi: 10.1371/journal.ppat.1003588

Mostowy, S., and Cossart, P. (2011). Septins as key regulators of actin based processes in bacterial infection. Biol. Chem. 392, 831-835. doi: 10.1515/BC.2011.078

Mostowy, S., and Cossart, P. (2012). Septins: the fourth component of the cytoskeleton. Nat. Rev. Mol. Cell Biol. 13, 183-194. doi: 10.1038/nrm3284

Mostowy, S., Danckaert, A., Tham, T. N., Machu, C., Guadagnini, S., PizarroCerdá, J., et al. (2009a). Septin 11 restricts InlB-mediated invasion by Listeria. J. Biol. Chem. 284, 11613-11621. doi: 10.1074/jbc.M900231200

Mostowy, S., Janel, S., Forestier, C., Roduit, C., Kasas, S., Pizarro-Cerdá, J., et al. (2011a). A role for septins in the interaction between the Listeria monocytogenes INVASION PROTEIN InlB and the Met receptor. Biophys. J. 100, 1949-1959. doi: 10.1016/j.bpj.2011.02.040

Mostowy, S., Nam Tham, T., Danckaert, A., Guadagnini, S., Boisson-Dupuis, S., Pizarro-Cerdá, J., et al. (2009b). Septins regulate bacterial entry into host cells. PLoS ONE 4:e4196. doi: 10.1371/journal.pone.0004196
Mostowy, S., Sancho-Shimizu, V., Hamon, M. A., Simeone, R., Brosch, R., Johansen, T., et al. (2011b). p62 and NDP52 proteins target intracytosolic Shigella and Listeria to different autophagy pathways. J. Biol. Chem. 286, 26987-26995. doi: 10.1074/jbc.M111.223610

Mostowy, S., and Shenoy, A. R. (2015). The cytoskeleton in cell-autonomous immunity: structural determinants of host defence. Nat. Rev. Immunol. 15, 559-573. doi: $10.1038 /$ nri3877

Nölke, T., Schwan, C., Lehmann, F., Østevold, K., Pertz, O., and Aktories, K. (2016). Septins guide microtubule protrusions induced by actindepolymerizing toxins like Clostridium difficile transferase (CDT). Proc. Natl. Acad. Sci. U.S.A. 113, 7870-7875. doi: 10.1073/pnas.1522717113

Ostrowski, P. P., Grinstein, S., and Freeman, S. A. (2016). Diffusion barriers, mechanical forces, and the biophysics of phagocytosis. Dev. Cell 38, 135-146. doi: 10.1016/j.devcel.2016.06.023

Pagliuso, A., Tham, T. N., Stevens, J. K., Lagache, T., Persson, R., Salles, A., et al. (2016). A role for septin 2 in Drp1-mediated mitochondrial fission. EMBO Rep. 17, 858-873. doi: $10.15252 / \mathrm{embr} .201541612$

Pizarro-Cerdá, J., Jonquières, R., Gouin, E., Vandekerckhove, J., Garin, J., and Cossart, P. (2002). Distinct protein patterns associated with Listeria monocytogenes InlA- or InlB-phagosomes. Cell. Microbiol. 4, 101-115. doi: 10.1046/j.1462-5822.2002.00169.x

Pizarro-Cerdá, J., Kühbacher, A., and Cossart, P. (2012). Entry of Listeria monocytogenes in mammalian epithelial cells: an updated view. Cold Spring Harb. Perspect. Med. 2:a010009. doi: 10.1101/cshperspect.a010009

Ray, K., Marteyn, B., Sansonetti, P. J., and Tang, C. M. (2009). Life on the inside: the intracellular lifestyle of cytosolic bacteria. Nat. Rev. Microbiol. 7, 333-340. doi: $10.1038 /$ nrmicro2112

Rodríguez-Escudero, I., Hardwidge, P. R., Nombela, C., Cid, V. J., Finlay, B. B., and Molina, M. (2005). Enteropathogenic Escherichia coli type III effectors alter cytoskeletal function and signalling in Saccharomyces cerevisiae. Microbiology 151, 2933-2945. doi: 10.1099/mic.0.28072-0

Rogers, L. D., Brown, N. F., Fang, Y., Pelech, S., and Foster, L. J. (2011). Phosphoproteomic analysis of Salmonella-infected cells identifies key kinase regulators and SopB-dependent host phosphorylation events. Sci. Signal. 4:rs9. doi: 10.1126/scisignal.2001668

Schmutz, C., Ahrne, E., Kasper, C. A., Tschon, T., Sorg, I., Dreier, R. F., et al. (2013). Systems-level overview of host protein phosphorylation during Shigella flexneri infection revealed by phosphoproteomics. Mol. Cell. Proteomics 12, 2952-2968. doi: 10.1074/mcp.M113.029918

Scholz, R., Imami, K., Scott, N. E., Trimble, W. S., Foster, L. J., and Finlay, B. B. (2015). Novel host proteins and signaling pathways in enteropathogenic E. coli pathogenesis identified by global phosphoproteome analysis. Mol. Cell Proteomics 14, 1927-1945. doi: 10.1074/mcp.M114.046847

Schwan, C., Kruppke, A. S., Nölke, T., Schumacher, L., Koch-Nolte, F., Kudryashev, M., et al. (2014). Clostridium difficile toxin CDT hijacks microtubule organization and reroutes vesicle traffic to increase pathogen adherence. Proc. Natl. Acad. Sci. U.S.A. 111, 2313-2318. doi: 10.1073/pnas.1311589111

Sirianni, A., Krokowski, S., Lobato-Márquez, D., Buranyi, S., Pfanzelter, J., Galea, D., et al. (2016). Mitochondria mediate septin cage assembly to promote autophagy of Shigella. EMBO Rep. 17, 1029-1043. doi: $10.15252 / \mathrm{embr} .201541832$

Stavru, F., Bouillaud, F., Sartori, A., Ricquier, D., and Cossart, P. (2011). Listeria monocytogenes transiently alters mitochondrial dynamics during infection. Proc. Natl. Acad. Sci. U.S.A. 108, 3612-3617. doi: 10.1073/pnas.1100126108

Takizawa, P. A., Derisi, J. L., Wilhelm, J. E., and Vale, R. D. (2000). Plasma membrane compartmentalization in yeast by messenger RNA transport and a septin diffusion barrier. Science 290, 341-344. doi: $10.1126 /$ science. 290.5490 .341

Tanaka-Takiguchi, Y., Kinoshita, M., and Takiguchi, K. (2009). Septin-mediated uniform bracing of phospholipid membranes. Curr. Biol. 19, 140-145. doi: 10.1016/j.cub.2008.12.030

van der Vaart, M., Korbee, C. J., Lamers, G. E., Tengeler, A. C., Hosseini, R., Haks, M. C., et al. (2014). The DNA damage-regulated autophagy modulator DRAM1 links mycobacterial recognition via TLR-MYD88 to autophagic defense [corrected]. Cell Host Microbe 15, 753-767. doi: 10.1016/j.chom.2014. 05.005

Volceanov, L., Herbst, K., Biniossek, M., Schilling, O., Haller, D., Nölke, T., et al. (2014). Septins arrange F-actin-containing fibers on the Chlamydia trachomatis 
inclusion and are required for normal release of the inclusion by extrusion. MBio 5, e01802-01814. doi: 10.1128/mBio.01802-14

Weiner, A., Mellouk, N., Lopez-Montero, N., Chang, Y. Y., Souque, C., Schmitt, C., et al. (2016). Macropinosomes are key players in early shigella invasion and vacuolar escape in epithelial cells. PLoS Pathog. 12:e1005602. doi: 10.1371/journal.ppat.1005602

Welch, M. D., and Way, M. (2013). Arp2/3-mediated actin-based motility: a tail of pathogen abuse. Cell Host Microbe 14, 242-255. doi: 10.1016/j.chom.2013.08.011

Willis, A., Mazon-Moya, M., and Mostowy, S. (2016). Investigation of septin biology in vivo using zebrafish. Methods Cell Biol. 136, 221-241. doi: 10.1016/bs.mcb.2016.03.019

Yoshikawa, Y., Ogawa, M., Hain, T., Yoshida, M., Fukumatsu, M., Kim, M., et al. (2009). Listeria monocytogenes ActA-mediated escape from autophagic recognition. Nat. Cell Biol. 11, 1233-1240. doi: 10.1038/ ncb1967

Conflict of Interest Statement: The authors declare that the research was conducted in the absence of any commercial or financial relationships that could be construed as a potential conflict of interest.

Copyright $\odot 2016$ Torraca and Mostowy. This is an open-access article distributed under the terms of the Creative Commons Attribution License (CC BY). The use, distribution or reproduction in other forums is permitted, provided the original author(s) or licensor are credited and that the original publication in this journal is cited, in accordance with accepted academic practice. No use, distribution or reproduction is permitted which does not comply with these terms. 